\title{
Material removal mechanism in rotary in-feed grinding - Modeling and analysis -
}

\author{
Wentong LU*, Libo ZHOU*, Jun SHIMIZU*, Teppei ONUKI*, \\ Hirotaka OJIMA* and Takeyuki YAMAMOTO** \\ *Graduate School of Science and Engineering, Ibaraki University \\ 4-12-1 Nakanarusawa, Hitachi, Ibaraki 316-8511, Japan \\ E-mail: 16nd209a@vc.ibaraki.ac.jp \\ ${ }^{* *}$ School of Engineering, Ibaraki University \\ 4-12-1 Nakanarusawa, Hitachi, Ibaraki 316-8511, Japan
}

Received: 10 July 2020; Revised: 22 August 2020; Accepted: 16 September 2020

\begin{abstract}
The rotary in-feed grinding is the most promising process to ensure the controllability of wafer geometry including the thickness and flatness. This makes it be widely applied into manufacturing of mono-crystal wafers, such as silicon, silicon carbide, sapphire, lithium tantalate, and etc. Unlike the conventional surface grinding, however, the rotary in-feed grinding is a complex material removal operation in which the extent of interaction between the abrasives and wafer keeps varying during the wheel/wafer contact zone. Such characteristic property significantly influences the chip formation, the homogeneousness of surface topography, the variations in grinding forces and the power consumption in the radial direction of the wafer. In this paper, the grinding mechanisms including the geometry, statics and surface topography were theoretically analyzed on a proposed grinding model and organized as follows. Described in Section 2 is the geometric analysis which mathematically expresses the wafer profile and a variety of information regarding to the dimensions of chip formed. The results suggest that the chip cross section increases proportionally as increasing in the wafer radial distance. Based on the results of chip formation, the grinding forces respectively exerted on an individual abrasive, a single wheel segment and whole wafer are calculated in the Section 3. Also, the necessary grinding power and generated grinding heat are estimated by statics analysis. In the Section 4 , the surface roughness and its variation in a radial direction of the wafer are then derived by taking into consideration of the distribution of abrasive protrusion in height-wise.
\end{abstract}

Keywords : Rotary in-feed grinding, Cutting path, Wafer profile, Chip formation, Grinding force, Topography, Surface roughness

\section{Introduction}

Mono-crystal materials, such as sapphire, silicon carbide and lithium tantalate are of great interest in the fields of microelectronics, optics and aerospace, due to their extraordinary functionality. Most of electronic and semiconductor devices in our daily lives are built on the substrates or wafers of those functional materials (Pishchik et al., 2009; Gerhardt et al., 2011; Strukov et al., 1998). Mono-crystal ingots normally undergo several manufacturing processes including slicing, beveling, grinding, lapping, polishing and etching, to be shaped into wafers (Tönshoff et al., 1990). The rotary in-feed grinding is the most promising process to ensure the controllability of wafer geometry including the thickness and flatness (King and Hahn., 1986). Wafer profile, surface roughness and subsurface damage are three major indicators to evaluate the performance of rotary in-feed grinding. Over the past years, numerous efforts have been invested to achieve better wafer profile (ex. less total thickness variation), better topography (ex. less surface roughness) and less subsurface damage.

In rotary in-feed grinding, both wafer and underneath chuck are ground into a conic shape by slightly tilting the wheel rotational axis against the wafer rotational axis, to ensure the contact length between the grinding wheel and the 
wafer to be constant. Sun et al. proposed the machine configurations for the spindle axis adjustments (Sun et al., 2005), in which an important criterion is given to tilt the wheel rotation axis relative to the wafer rotation axis. Via the kinematic analysis and experiments, Tso et al. also stated that the inclination of grinding wheel axis must be set appropriately to improve the total thickness variation (TTV) of the ground substrate (Tso and Teng, 2001). In the report of (Zhang et al., 2006), Zhang et al. reviewed the hypothesis for the generation mechanisms of central dimples. Zhou et al. kinematically analyzed the cutting path of individual abrasive across the wafer surface to get the wafer profiles for different conbination of tilt angles of the wheel axis against the wafer axis (Zhou et al., 2003).

Regrading to the surface roughness, Tomita and Eda developed a fixed abrasive grinding wheel for producing an ultra-fine surface on a magnetic disk substrate (Tomita et al., 1996). Liu et al. investigated the effects of different abrasive shapes and dressing to predict surface roughness in grinding using kinematic simulations (Liu et al., 2013). Our previous study (Zhou et al., 2017) has theoretically analyzed the effects of grain size variation on the wafer surface topography. The study found that the actual surface roughness could become several folds larger than the theoretical prediction.

To reduce the subsurface damage (or SSD) induced in grinding, many approaches have been made from the viewpoints of grinding wheel specifications, grinding conditions and wafer properties. Lundt et al. stated that grinding of silicon wafer would cause unavoidable SSD (Lundt et al., 1994). Pei et al. found that the depth of subsurface crack on ground silicon wafers is approximately equal to half of the diamond abrasive size used in the grinding wheel (Pei et al., 1999). Zhang et al. analytically predicted the depth of SSD in silicon wafer induced in the rotary in-feed grinding process (Zhang et al., 2019). This research stated that less SSD can be achieved with lower feed rate, lower wafer rotation speed and higher grinding wheel speed.

In order to associate the grinding conditions with the grinding results mentioned above, study on the material removal mechanism is very essential. In grinding mono-crystal materials, material removal in either ductile or brittle mode is one of the most interested issues (Doi et al., 2014). In order to realize ductile mode grinding, the depth of cut of each abrasive must be controlled to be less than the critical depth of cut (or DOC) of ground wafers. Young et al. derived the critical DOC for silicon wafers by the experimental investigation (Young et al., 2006, 2007). Our previous study showed that a better surface roughness can be achieved if the abrasive cutting depth is less than the critical DOC (Zhou et al., 2012). The study also indicated that the transition between the ductile and brittle modes can be controlled by the grinding conditions such as wheel rotational speed, wafer rotational speed and in-feed rate. Moreover, Lin et al. proposed a model to predict abrasive depth of cut in rotary in-feed grinding (Lin et al., 2018). It is claimed that the elastic-plastic behaviors in both wheel and wafer should be taken into consideration for accurately predicting the grain depth-of-cut in ultra-fine grinding. Therefore, the chip formation is a key for understanding the mono-crystal material removal mechanism. Unlike the conventional surface grinding, however, the rotary in-feed grinding is a complex material removal operation in which the extent of interaction between the abrasives and wafer keeps varying during the wheel/wafer contact zone. A further study is required to look insight into the variation in chip formation along the wafer radial direction.

The grinding force is another important issue to understand the interaction between the abrasives and the materials to be removed, and the induced subsurface damage. Couey et al. have developed a device for in-process force monitoring in precision grinding of silicon wafers (Couey et al., 2005). The research also indicated that the grinding force was influenced by the in-feed rate. Sun et al. provided a predictive model of grinding force in silicon wafer grinding, which corelated the grinding conditions to the grinding force and was validated by experimental results (Sun et al., 2016). A wireless dynamometer has been developed for rotary in-feed grinding in our previous study (Ishibashi et al., 2019). It has found that the grinding force gradually grew up when the wheel segment started to engage from the wafer center, and rapidly dropped to zero when the wheel segment exits from the wafer fringe. Therefore, a thorough analysis on the relationships between the chip formation and grinding statics becomes essential.

Based on the rotary in-feed grinding dynamics, in this paper, we mathematically analyze the cutting path of abrasive in 3D manner to obtain the wafer profile and chip formation along the cutting path. The grinding force exerted on an individual abrasive is corelated to the chip cross section, which is then extended to a single wheel segment and whole wafer. Also, the consumed grinding power and generated grinding heat are estimated for such grinding dynamics. In the rest sections of this paper, the number of effective cutting edge is estimated by taking into consideration of the distribution of abrasive protrusion in height-wise, to derive the resultant roughness on the wafer surface and its variation in a radial direction of the wafer. 


\section{Geometric analysis}

\subsection{Rotary in-feed grinding model}

The rotary in-feed grinding model is illustrated in Fig.1. A wafer is mounted via a porous vacuum chuck and a cup-type grinding wheel is placed opposite with a tilt angle $\alpha$ and an offset equivalent to the wheel radius $R_{1}$. During grinding, both grinding wheel and wafer rotate around their own axes simultaneously while the wheel is fed into the wafer with a given in-feed rate $f$. Such in-feed grinding dynamics can keep the contact area unchanged and thereby to deliver a stable grinding performance throughout the grinding process. The symbols used in model description are listed in Table 1, where the common parameters used in wheel and wafer specifications and grinding conditions are also specified for the subsequent calculation. Listed in Table 2 are symbols used in model analysis and evaluation.

In a Cartesian coordinate system with the origin fixed at the wafer center, our previous study (Zhou et al., 2003) has found out the mathematical description of the cutting path for an abrasive to travel across the wafer surface in 2D space. Taking the tilt angles into the consideration, the cutting path description is then updated to 3D space and given in a matrix form as Eq. (1).

$$
\left[\begin{array}{c}
x(\tau) \\
y(\tau) \\
z(\tau) \\
1
\end{array}\right]=\boldsymbol{A} \cdot \boldsymbol{B} \cdot \boldsymbol{C} \cdot \boldsymbol{D} \cdot \boldsymbol{E} \cdot \boldsymbol{F}\left[\begin{array}{c}
x_{g} \\
y_{g} \\
z_{g} \\
1
\end{array}\right]
$$

where $\left(x_{g}, y_{g}, z_{g}\right)$ is the initial position of corresponding abrasive and $\tau$ is the grinding time. The matrices $\boldsymbol{A}$ and $\boldsymbol{E}$ represent the rotations of wafer and wheel respectively, and are specified as;
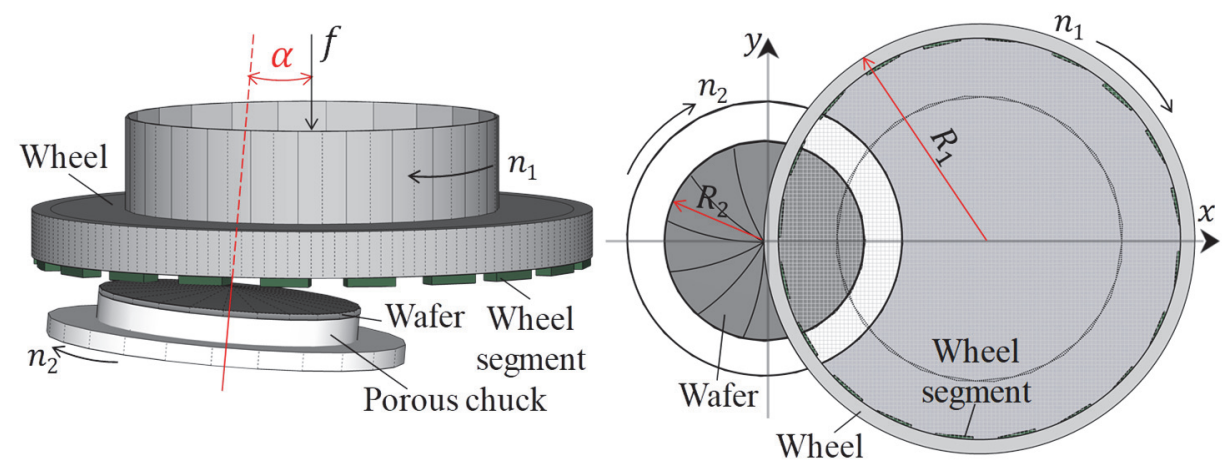

Fig.1 Rotary infeed grinding.

Table 1 The symbols used in model description and parameters for specifications and conditions.

\begin{tabular}{ccllc}
\hline \hline & $R_{1}$ & Wheel radius & 150 & {$[\mathrm{rpm}]$} \\
& $l$ & Wheel segment length & 20 & {$[\mathrm{~mm}]$} \\
Wheel & $w$ & Wheel segment width & 3 & {$[\mathrm{~mm}]$} \\
specifications & $r_{g}$ & Abrasive radius $\left(r_{0}, m \sigma\right)$ & $(6,1.5)$ for \#1000 & {$[\mu \mathrm{mm}]$} \\
& $m$ & Cut-off coefficient & 1 & - \\
& $V_{g}$ & Abrasive concentration & 25 & {$[\mathrm{vol} \%]$} \\
& $2 \gamma$ & Abrasive vertex angle & $165 \quad($ Syoji, 2004) & {$\left[{ }^{\circ}\right]$} \\
\hline Wafer & $R_{2}$ & Wafer radius & $100 \sim 150$ & {$[\mathrm{~mm}]$} \\
specifications & $C p$ & Specific grinding energy & $8600($ for Si) & {$[\mathrm{MPa}]$} \\
\hline \multirow{6}{*}{ Grinding } & $n_{1}$ & Wheel rotational speed & $1000 \sim 5000$ & {$[\mathrm{rpm}]$} \\
conditions & $n_{2}$ & Wafer rotational speed & $25 \sim 100$ & {$[\mathrm{rpm}]$} \\
& $f$ & Feed rate & $0.01 \sim 0.1$ & {$[\mathrm{~mm} / \mathrm{min}]$} \\
& $\alpha$ & Tilt angle of wafer axis & $6.5 \times 10^{-4}$ & {$\left[{ }^{\circ}\right]$} \\
\hline
\end{tabular}


Lu, Zhou, Shimizu, Onuki, Ojima and Yamamoto,

Journal of Advanced Mechanical Design, Systems, and Manufacturing, Vol.14, No.6 (2020)

Table 2 The symbols used in model evaluation.

\begin{tabular}{clc}
\hline$r_{2}$ & Wafer radial distance & {$[\mathrm{mm}]$} \\
$\Delta$ & Cutting depth & {$[\mathrm{mm}]$} \\
$S_{2}$ & Side area of ground wafer & {$\left[\mathrm{mm}^{2}\right]$} \\
$N_{e}$ & Density of effective cutting edge & {$\left[\mathrm{partical} / \mathrm{mm}^{2}\right]$} \\
$a_{g}$ & Size of chip cross section & {$\left[\mathrm{mm}^{2}\right]$} \\
$q_{g}$ & Chip volume & {$\left[\mathrm{mm}^{3}\right]$} \\
$f_{t}$ & Grinding force on an individual abrasive & {$[\mathrm{N}]$} \\
$F_{t}$ & Grinding force on a wheel segment & {$[\mathrm{N}]$} \\
$\Sigma F_{t}$ & Total grinding force on the entire wafer & {$[\mathrm{N}]$} \\
$q$ & Power density & {$\left[\mathrm{W} / \mathrm{mm}^{2}\right]$} \\
$P$ & Grinding power & {$[\mathrm{W}]$} \\
$R_{z}$ & Surface roughness & {$[\mu \mathrm{m}]$} \\
\hline
\end{tabular}

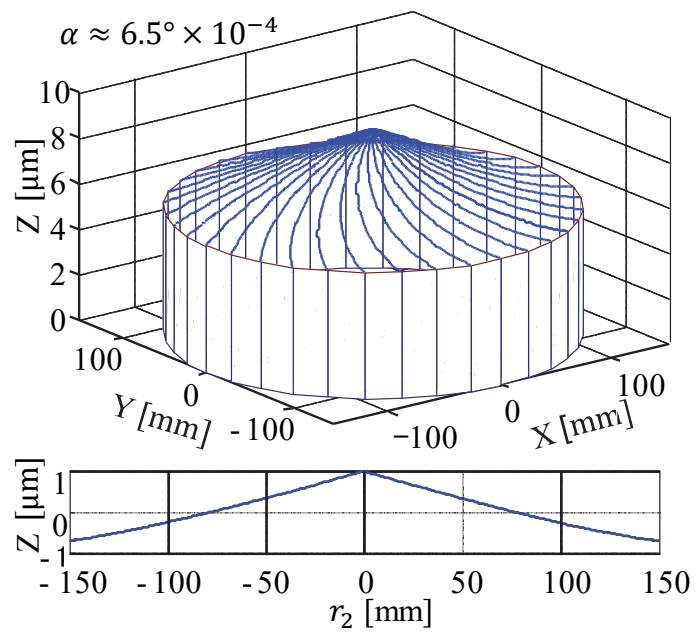

Fig.2 Cutting path and wafer profile

$$
\begin{aligned}
\boldsymbol{A} & =\left[\begin{array}{cccc}
\cos 2 \pi n_{2} \tau & \sin 2 \pi n_{2} \tau & 0 & 0 \\
-\sin 2 \pi n_{2} \tau & \cos 2 \pi n_{2} \tau & 0 & 0 \\
0 & 0 & 1 & 0 \\
0 & 0 & 0 & 1
\end{array}\right] \\
\boldsymbol{E} & =\left[\begin{array}{cccc}
\cos 2 \pi n_{1} \tau & -\sin 2 \pi n_{1} \tau & 0 & 0 \\
\sin 2 \pi n_{1} \tau & \cos 2 \pi n_{1} \tau & 0 & 0 \\
0 & 0 & -f \tau & 0 \\
0 & 0 & 0 & 1
\end{array}\right]
\end{aligned}
$$

while, the matrices $\boldsymbol{B}$ and $\boldsymbol{C}$ respectively express the tilt angles of wafer around X-axis and Y-axis, are given as;

$$
\begin{aligned}
\boldsymbol{B} & =\left[\begin{array}{cccc}
1 & 0 & 0 & 0 \\
0 & \cos \alpha & -\sin \alpha & 0 \\
0 & \sin \alpha & \cos \alpha & 0 \\
0 & 0 & 0 & 1
\end{array}\right] \\
\boldsymbol{C} & =\left[\begin{array}{cccc}
\cos \beta & 0 & \sin \beta & 0 \\
0 & 1 & 0 & 0 \\
-\sin \beta & 0 & \cos \beta & 0 \\
0 & 0 & 0 & 1
\end{array}\right]
\end{aligned}
$$

and, the matrices $\boldsymbol{D}$ and $\boldsymbol{F}$ are the translations of the offset $R_{1}$. 


$$
\begin{aligned}
\boldsymbol{D} & =\left[\begin{array}{lllc}
1 & 0 & 0 & R_{1} \\
0 & 1 & 0 & 0 \\
0 & 0 & 1 & 0 \\
0 & 0 & 0 & 1
\end{array}\right] \\
\boldsymbol{F} & =\left[\begin{array}{cccc}
1 & 0 & 0 & -R_{1} \\
0 & 1 & 0 & 0 \\
0 & 0 & 1 & 0 \\
0 & 0 & 0 & 1
\end{array}\right]
\end{aligned}
$$

Figure 2 shows a typical example of the cutting path in 3D manner, calculated at $\alpha \approx 6.5^{\circ} \times 10^{-4}, \beta=0^{\circ}$. From the group of these cutting paths, we are able to get the cross-sectional profile of the wafer as shown in the bottom panel of Fig.2. It is also found whenever $\alpha \neq 0^{\circ}$ that the wafer profile falls in a convex cone shape so that the wheel constantly makes a half-contact with the wafer surface during grinding, which is favored in most wafer manufacturing.

\subsection{Chip formation}

Figure 3 shows the engagement of the wheel against the wafer. The shadow band illustrated in (a) represents the volume removed in one rotation of the wheel, where the depth of cut is $\Delta=f / n_{2}$ and the travel length is $L=$ $2 \pi r_{2} n_{2} / n_{1}$. It is clear that the depth of cut is kept constant once the grinding conditions are given. Here, the wafer is considered as a collected concentric annulus with different radius $r_{2}$. One annulus positioned at $\left[r_{2}, r_{2}+\mathrm{d} r_{2}\right]$ is zoomed up in Fig. 3 (b) for the detailed study. The side area $S_{2}$ removed in one rotation of the wheel is expressed as Eq. (2).

$$
S_{2}=\Delta \cdot L=2 \pi \frac{f}{n_{1}} r_{2}=2 \pi \cdot \frac{n_{2}}{n_{1}} \cdot \Delta \cdot r_{2}
$$

As shown in Fig. 4, the side area $S_{2}$ is proportional to the wafer radial distance $r_{2}$, the depth of cut $\Delta$ and the rotational speed ratio $n_{2} / n_{1}$. It should be noted that the wafer rotational speed $n_{2}$ does not affect the size of $S_{2}$, but does affect its geometric shape. As illustrated in-panel in Fig. 4, increasing in $n_{2}$ leads to a long and thin shape of $S_{2}$ while keeps the area of $S_{2}$ unchanged.

According to the principle of continuity, $S_{2}$ is shared by the number of effective cutting edges $j=2 \pi R_{1} w \cdot N_{e}$ which are evenly distributed across the wheel working surface, where $w$ is the width of wheel segment, $N_{e}$ is the density of effective cutting edge (Zhou et al., 2017). Therefore, the chip cross section removed by an individual abrasive cutting edge at the wafer radial distance $r_{2}$ is given below.

$$
a_{g}=\frac{S_{2}}{j}=\frac{1}{R_{1} w N_{e}} \frac{f}{n_{1}} r_{2}=\frac{1}{R_{1} w N_{e}} \frac{n_{2}}{n_{1}} \Delta \cdot r_{2}
$$

Also, the chip volume $q_{g}$ and average chip cross section area $\bar{a}_{g}$ are then given below;

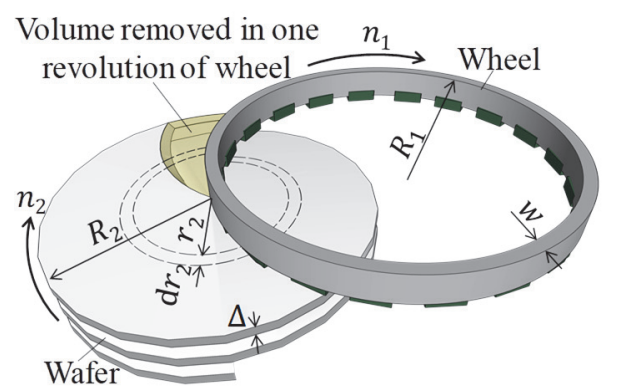

(a) Depth of cut and removed volume



(b) Side area in a ground annulus



(c) Cutting chip by individual cutting edge

Fig.3 Chip formation. 


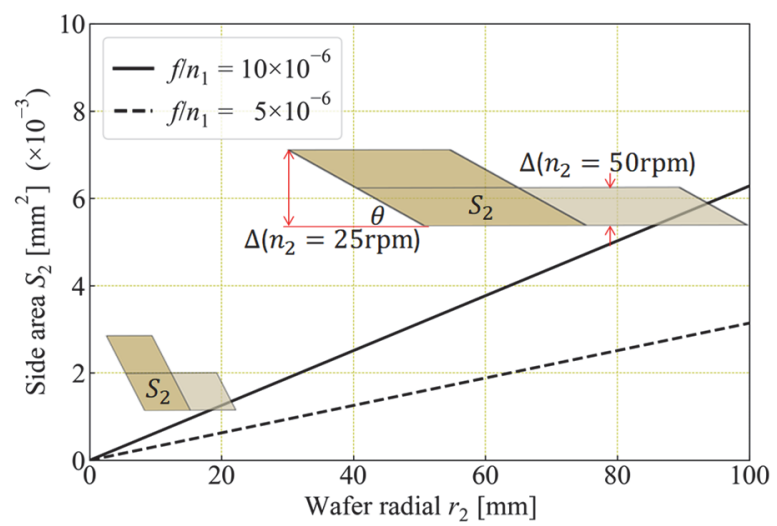

Fig.4 Side area of material removed and depth of cut.

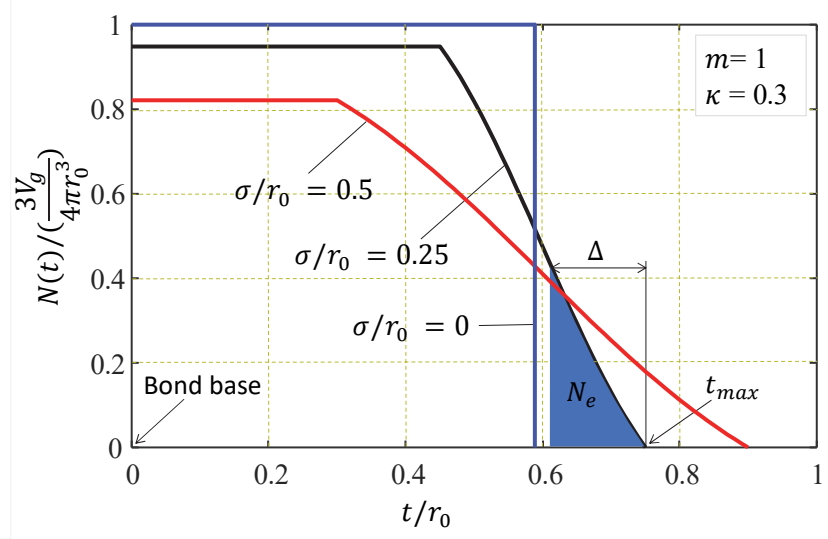

Fig.5 Protrusion height distribution and effective cutting Edge.

$$
\begin{aligned}
& q_{g}=\int_{0}^{R_{2}} a_{g} \mathrm{~d} r_{2}=\frac{1}{2 w N_{e}} \frac{R_{2}^{2}}{R_{1}} \cdot \frac{n_{2}}{n_{1}} \Delta \\
& \bar{a}_{g}=\frac{1}{R_{2}} \int_{0}^{R_{2}} a_{g} \mathrm{~d} r_{2}=\frac{1}{2 w N_{e}} \frac{R_{2}}{R_{1}} \cdot \frac{n_{2}}{n_{1}} \Delta
\end{aligned}
$$

As shown in Eq. (3) and Fig. 3 (c), the chip cross section $a_{g}$ proportionally increases as the abrasive goes from the wafer center to its outer periphery. Here, the effective cutting edge density $N_{e}$ is an important factor to determine the chip cross section $a_{g}$ and for the rest analysis. Our previous paper (Zhou et al., 2017) has theoretically studied the distribution of protrusion height $t$ of abrasives on the wheel working surface and expressed as below;

$$
N(t)=\left\{\begin{array}{lr}
\frac{3 V_{g}}{4 \pi r_{0}^{3}} \frac{1}{1+3\left[1-\frac{\sqrt{\frac{2}{\pi}} m \cdot \exp \left(-\frac{m^{2}}{2}\right)}{1-\operatorname{erfc}\left(\frac{m}{\sqrt{2}}\right)}\right]\left(\frac{\sigma}{r_{0}}\right)^{2}} & \left(0 \leq t<2 \kappa\left(r_{0}-m \sigma\right)\right) \\
\frac{3 V_{g}}{8 \pi r_{0}^{3}} \frac{1}{1+3\left[1-\frac{\sqrt{\frac{2}{\pi}} m \cdot \exp \left(-\frac{m^{2}}{2}\right)}{1-\operatorname{erfc}\left(\frac{m}{\sqrt{2}}\right)}\right]\left(\frac{\sigma}{r_{0}}\right)^{2}} \frac{\operatorname{erfc}\left(\frac{t-2 \kappa r_{0}}{2 \sqrt{2} \sigma \kappa}\right)-\operatorname{erfc}\left(\frac{m}{\sqrt{2}}\right)}{1-\operatorname{erfc}\left(\frac{m}{\sqrt{2}}\right)} & \left(t \geq 2 \kappa\left(r_{0}-m \sigma\right)\right)
\end{array}\right.
$$

where, the abrasive radius $r_{g}$ is assumed to follow a normal distribution $\left(r_{0}, \sigma\right)$ and truncated by $r_{0} \pm m \sigma$. The multiplier $m=1$ is favored by most wheel manufacturers. The abrasive drops out when its protrusion height $t$ exceeds $2 \kappa r_{g}$ from the bond base, where $\kappa$ takes $0 \leq \kappa<1$ and referred as the protruding coefficient. It is found out that the number of abrasives $N(t)$ are constant when the protrusion height ranges $0 \leq t<2 \kappa\left(r_{0}-m \sigma\right)$, then decreases as increasing in $t$ and eventually converges to zero at the outmost periphery of the wheel. Figure 5 shows the normalized variation of $N(t)$ for three kinds of wheels with different standard deviation $\sigma$ in the distribution of abrasive size.

Obviously, only those abrasives which have top protrusion possibly become effective to engage into material removal. The amount of these effective cutting edges can be calculated by integration of $N(t)$ within the range of $t_{\text {max }}-\Delta<$ $t<t_{\max }$ which is illustrated by a light shade in Fig. 5 and expressed in Eq. (7).

$$
N_{e}(\Delta)=\int_{t_{\max }-\Delta}^{t_{\max }} N(t) \mathrm{d} t
$$

where $t_{\max }=2 \kappa\left(r_{0}+m \sigma\right)$ for a given protrusion coefficient $\kappa$ and abrasive radius distribution $m \sigma$. For most rotary in-feed grinding as shown in Fig.1, $\Delta$ ranges from submicron to several microns, depending on the in-feed rate $f$ and the wafer rotational speed $n_{2}$. Therefore, only a fraction of abrasive at the top protrusions are possibly engaged in material removal and become effective cutting edges. For such a wheel $\left(R_{1}=150 \mathrm{~mm}, w=3 \mathrm{~mm}, V_{g}=\right.$ $\left.25 \mathrm{vol} \%, r_{0}=6 \mu \mathrm{m}, \sigma=1.5 \mu \mathrm{m}, \kappa=0.3\right)$, the cutting edge exposed on the working surface is about $1000 \mathrm{p} / \mathrm{mm}^{2}$. The 


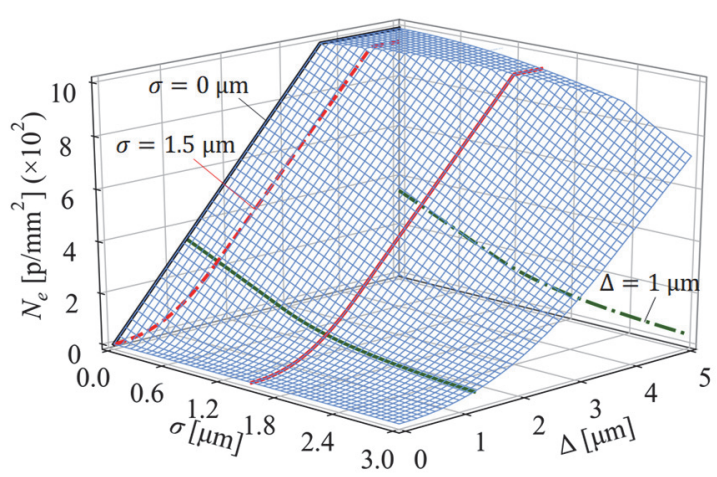

Fig. 6 Effective cutting edge density $N_{e}$.

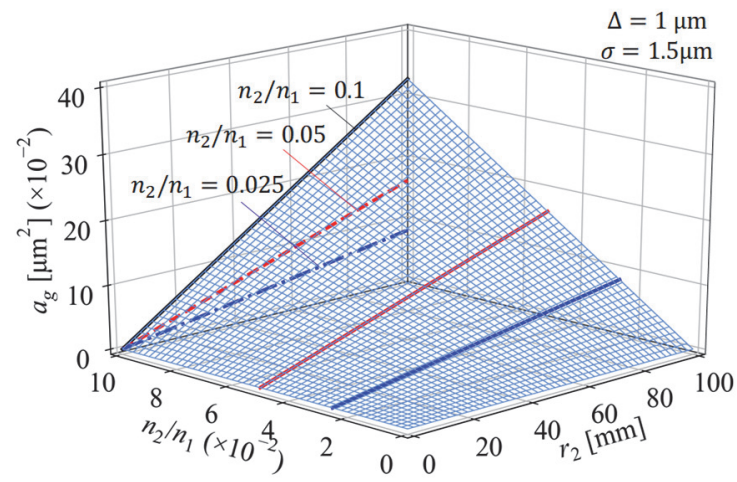

Fig.7 Cross sectional area of individual chip.

effective cutting edge density $N_{e}$ is calculated and shown in Fig. 6. Depending on the depth of cut $\Delta\left(=f / n_{2}\right)$ and standard deviation $\sigma$ in the distribution of abrasive size, $N_{e}$ ranges $20 \sim 200 \mathrm{p} / \mathrm{mm}^{2}$, or about $2 \% \sim 20 \%$ of the total.

Using the above $N_{e}$, the chip cross section area $a_{g}$ is calculated as a function of $\left(r_{2}, n_{2} / n_{1}\right)$. The results for $\Delta=$ $1 \mu \mathrm{m}$ is shown in Fig. 7. The chip cross section by an individual abrasive cutting edge increases linearly with an increasing in both rotational speed ratio $n_{2} / n_{1}$ and wafer radial distance $r_{2}$. Choosing a high wheel speed effectively constrains the chip cross section.

\section{Statics analysis}

\subsection{Grinding force}

When an abrasive is engaged in material removal, as illustrated in Fig. 8, its cutting force in tangential direction (along cutting path) is the product of the specific removal energy $C p$ and the chip cross section $a_{g}$. Therefore, based on the Eq. (3), the tangential force on an individual abrasive is expressed as Eq. (8).

$$
f_{t}=C p \cdot a_{g}=\frac{C p}{R_{1} w N_{e}} \cdot \frac{n_{2}}{n_{1}} \Delta \cdot r_{2}
$$

Here, Eq. (8) addresses that the tangential force $f_{t}$ not only is effected by the grinding conditions $n_{2} / n_{1}, \Delta$, but also by the wheel specifications $R_{1}, w, N_{e}$, and the cutting position $r_{2}$ as well. Figure 9 plots two sets of tangential force $f_{t}$ appearing at different wheel rotational speed $n_{1}$, as a function of the grinding time $\tau$. It is found that $f_{t}$ is halved when the wheel speed $n_{1}$ is doubled. The iteration of $f_{t}$ is also doubled in frequency. It should be point out that the specific removal energy $C p$ depends on not only the property of wafer material but also the size of $a_{g}$. A smaller $a_{g}$ normally results in relatively larger $C p$ because of the "size effect"

Within the annulus positioned at $\left[r_{2}, r_{2}+\mathrm{d} r_{2}\right]$ mentioned above, the sum of number of abrasives totally engaged is

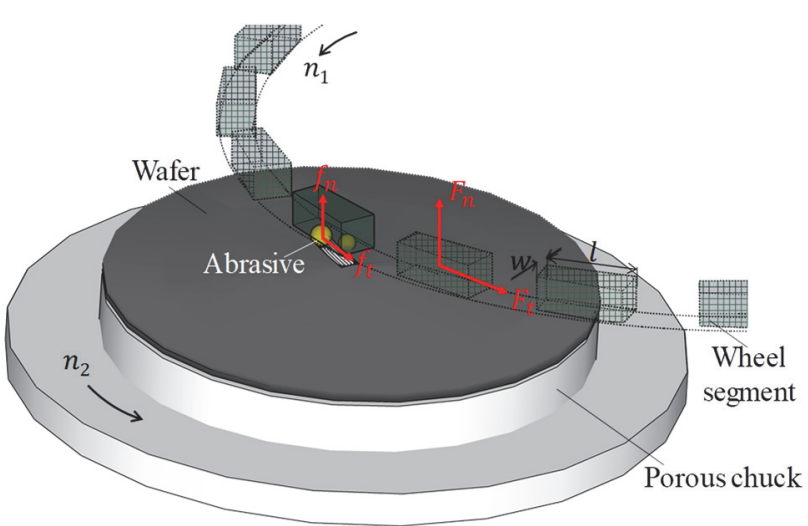

Fig.8 Illustration of grinding force components.

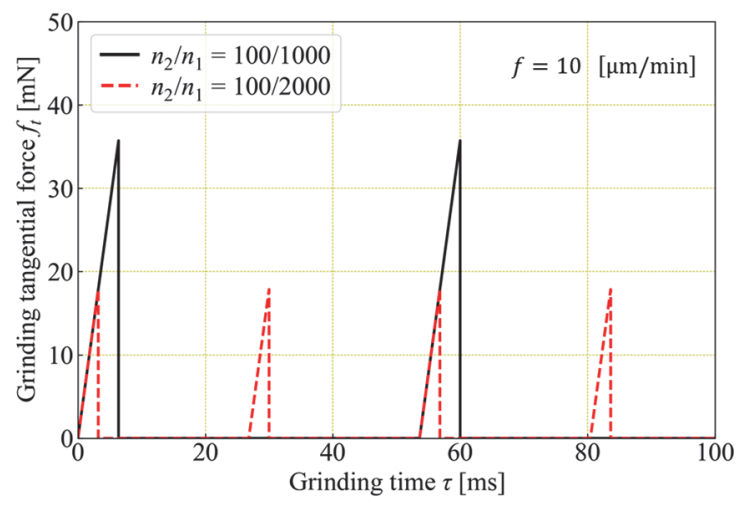

Fig.9 Grinding force on an individual abrasive. 
given as $N_{e} \cdot w \cdot \mathrm{d} r_{2}$. Thus, the tangential grinding force on a wheel segment is calculated as Eq. (9).

$$
F_{t}=\int_{r_{2}-l / 2}^{r_{2}+l / 2} f_{t} \cdot N_{e} w \mathrm{~d} r_{2}=C p \frac{l}{R_{1}} \cdot \frac{n_{2}}{n_{1}} \Delta \cdot r_{2}
$$

where, $l$ is the length of a wheel segment. $\quad F_{t}$ is found to be proportional to the segment length $l$, speed ratio $n_{2} / n_{1}$ and the depth of cut $\Delta$. By taking the effect at the wafer center and fringe, where the wheel segment is not fully contacted with the wafer, into consideration, variation of the tangential grinding force on a wheel segment $F_{t}$ is shown in Fig. 10. $\quad F_{t}$ gradually grows along the wafer radial distance $r_{2}$, and rapidly drops to zero when the wheel segment exits from the wafer fringe. This finding matches well with our previous experiments on the silicon wafer grinding (Ishibashi et al., 2019). This fact also suggests that the wheel rotational direction is important for wafer thinning process to ensure the wheel segment traveling from the wafer center toward its fringe. Otherwise, the wafer may encounter a sudden increase in grinding force and highly risk in edge cracking.

\subsection{Grinding power and grinding heat}

The total grinding force $\sum F_{t}$ generated on the entire wafer surface is calculated by integration of $F_{t}$ within the range of $\left[0, R_{2}\right]$ which is expressed in Eq. (10).

$$
\sum F_{t}=\int_{0}^{R_{2}} f_{t} \cdot N_{e} w \mathrm{~d} r_{2}=\frac{C p}{2} \cdot \frac{R_{2}^{2}}{R_{1}} \cdot \frac{n_{2}}{n_{1}} \Delta
$$

The consumed grinding power is then the product of $\sum F_{t}$ and the wheel rotational velocity, expressed as;

$$
P=\sum F_{t} \cdot 2 \pi R_{1} n_{1}=C p \cdot \pi R_{2}^{2} \cdot f
$$

Both the total grinding force $\sum F_{t}$ (the solid line in Fig. 11) and the consumed grinding power $P$ (the broken line in Fig. 11) are proportional to the square of the wafer size $R_{2}$. Hence, it is necessary to pay attention to the rigidity and the spindle power of the grinding machine when grinding large diameter wafers.

Back to the Fig. 3 (b), the grinding power consumed on the annulus positioned at $\left[r_{2}, r_{2}+\mathrm{d} r_{2}\right]$ is expressed as;

$$
\mathrm{d} P=2 \pi R_{1} n_{1} \cdot f_{t} \cdot N_{e} w \mathrm{~d} r_{2}=2 \pi C p \cdot f \cdot r_{2} \mathrm{~d} r_{2}
$$

The corresponding surface area ground on the annulus is given as $2 \pi r_{2} \cdot n_{2} \cdot \mathrm{d} r_{2}$. The power density $q$ (or, the energy consumed on a specific area of the wafer per unit time) is then resulted as;

$$
q=\frac{\mathrm{d} P}{2 \pi r_{2} \cdot n_{2} \cdot \mathrm{d} r_{2}}=C p \cdot \frac{f}{n_{2}}=C p \cdot \Delta
$$

The power density is the source of the grinding heat and directly associated with the temperature rise in the wafer. It is found that the power density is consistent across the entire wafer, without depending on the radial distance $r_{2}$. The temperature distribution, however, may not be homogeneous due to the concentricity of round shape of the wafer. The details will be evaluated by use of FEM and reported in our subsequent study.

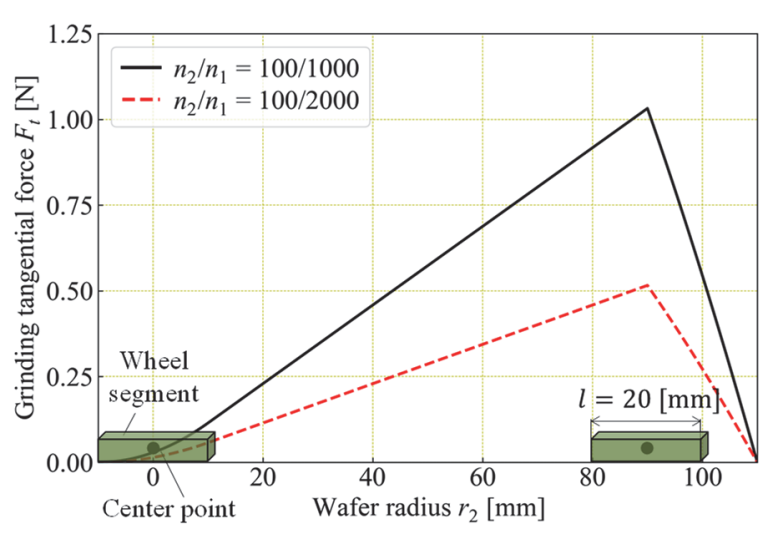

Fig.10 Grinding force on a wheel segment.

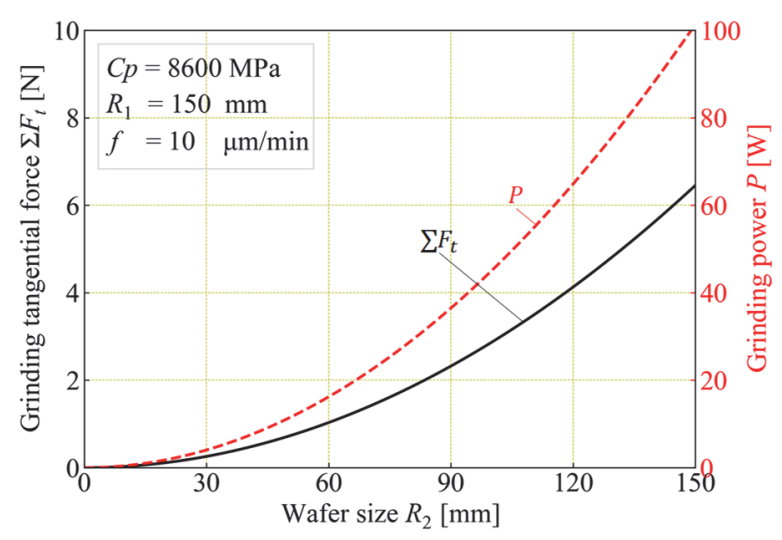

Fig.11 Total grinding force and grinding power. 


\section{Topography analysis}

Illustrated in Fig.12 is the surface topography generated on a ground wafer surface. Assuming the vertex angle of an abrasive is $2 \gamma$, the penetration depth of the abrasive into the wafer can be easily derived once the chip cross section $a_{g}$ is known.

$$
\frac{1}{2} R_{z} \cdot 2 R_{z} \tan \gamma=a_{g}
$$

Substituting Eq. (3) and (7) into the above equation, the surface roughness can be solved as;

$$
R_{Z}=\sqrt{\frac{1}{\tan \gamma R_{1} w} \cdot \frac{n_{2}}{n_{1}} \cdot \frac{\Delta}{N_{e}} \cdot r_{2}}
$$

It must be pointed out that $R_{z}$ in Eq. (14) is governed by the cross section $a_{g}$, while $a_{g}$ in Eq. (3) is the averaged cross section at the radial distance $r_{2}$ without counting the variation in the abrasive radius and protrusion height. Therefor, $R_{z}$ in Eq. (15) is a sort of equalized maximum surface roughness, smaller than that defined by ISO.

For such triangularly waved topography, the mean line roughness average $R_{a}$ is given as;

$$
R_{a}=\frac{1}{L}\left(\frac{1}{2} L \cdot \frac{R_{z}}{2}\right)=\frac{1}{4} R_{z}
$$

where, $L$ is the evaluation length. At the wafer center where the wafer radial distance $r_{2} \rightarrow 0$, in addition, $\mathrm{K}$. Ono suggested that the surface roughness should take its ultimate limit (Ono, 1962). The surface roughness $R_{Z}$ calculated at $\Delta=1 \mu \mathrm{m}$ is plotted in Fig. 13, as a function of $\left(r_{2}, n_{2} / n_{1}\right)$. The results suggest that the surface roughness becomes larger at the outer circumference of the wafer when $r_{2}$ approaches to $R_{2}$, but smaller as decreasing in the rotational speed ratio $n_{2} / n_{1}$. The trend matches well with the experimental results on silicon wafers in our previous report (Ebina et al., 2018). Figure 14 shows the surface roughness $R_{z}$ calculated at $r_{2}=50 \mathrm{~mm}$ while $\Delta$ and $n_{2} / n_{1}$ varies. The

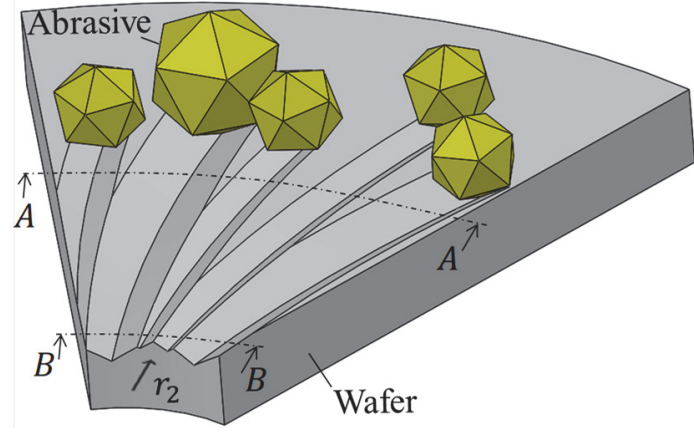

(a) the ground wafer surface

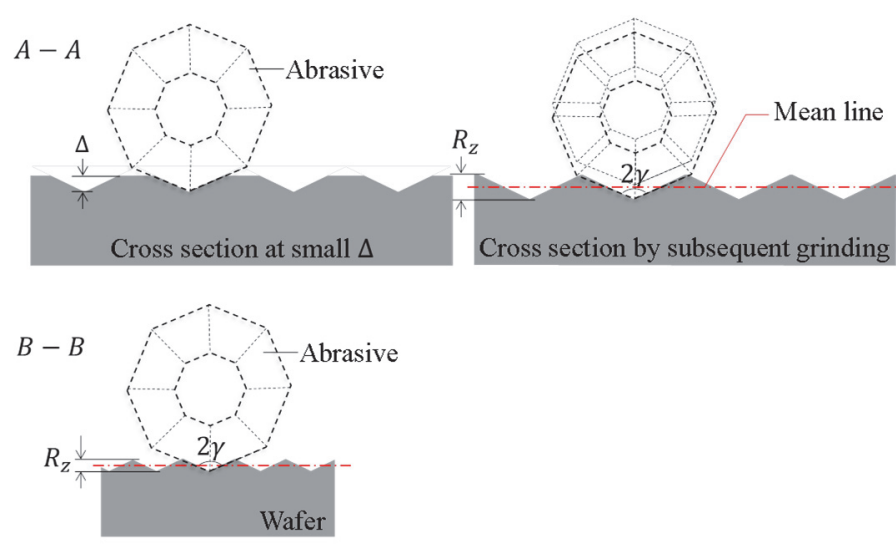

(b) the cross section of grinding path

Fig. 12 Illustration of the surface topography.

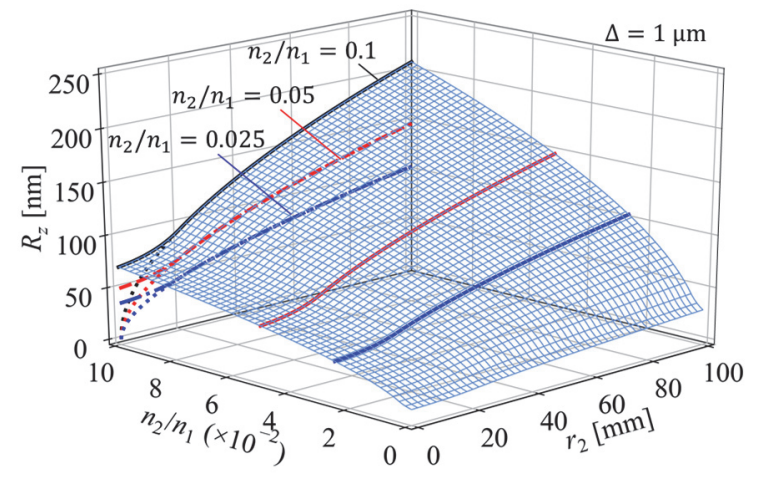

Fig.13 Effect of $n_{2} / n_{1}$ and $r_{2}$ on $R_{z}$

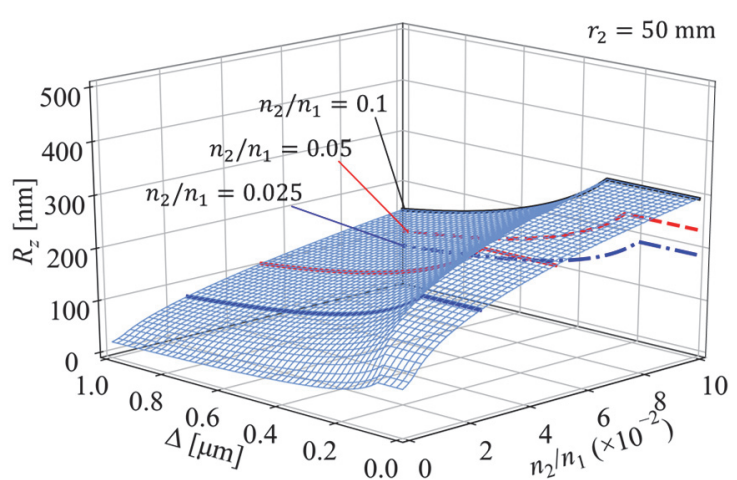

Fig.14 Effect of $n_{2} / n_{1}$ and $\Delta$ on $R_{z}$ 
results tell that inadequate depth of cut $\Delta$ may not improve the surface roughness due to the insufficient effective cutting edge (Fig. 6). Especially when $\Delta$ is extremely small $(\Delta<0.2 \mu \mathrm{m}$ for the current condition), as illustrated in Fig.12 (b), the wafer surface may not be completely removed in one rotation of the grinding wheel. The subsequent grinding still makes the surface roughness remain relatively high. Finally, it should also be noted that the Eq. (15) does not include the elastic recovery of the wafer after grinding, which could counter up to $40 \% \sim 60 \%$ of the depth of cut according to (Huang et al., 2020).

\section{Conclusions}

In this paper, the grinding mechanisms including the geometry, statics and surface topography were theoretically analyzed for the rotary in-feed grinding dynamics. The obtained results can be summarized as below:

1) The cutting path was mathematically solved in $3 \mathrm{D}$ manner to incorporate the tilt angles of wheel rotational axis against the wafer rotational axis, which is able to provide not only the trajectory of each abrasive but also the profile of ground wafers.

2) The chip cross section and chip volume were given along the cutting path to understand the chip formation associated with grinding conditions and wheel specifications.

3) The effective cutting edge density was estimated by taking into consideration of the distribution of abrasive protrusion in height-wise, which helps to precisely predict the chip cross section, grinding forces and surface roughness.

4) The grinding force on a wheel segment was proportional to the segment length, gradually grew along the wafer radial distance, and rapidly dropped to zero when the wheel segment exited from the wafer fringe.

5) Both total grinding force and consumed grinding power were found to be proportional to the square of the wafer size. This result suggested the rigidity and the spindle power of the grinding machine were important when grinding large diameter wafers.

6) The power density which was directly associated with the temperature rise during grinding was consistent across the entire wafer, without depending on the radial distance.

7) The surface roughness and its variation in a radial direction of the wafer were derived and discussed on varying grinding conditions. The depth of cut should be adequate to ensure sufficient cutting edge being effective.

\section{Acknowledgement}

The authors would like to deeply appreciate the support from the JSPS (Japan Society for the Promotion of Science) KAKENHI, Grant-in-Aid for Scientific Research (A) (No. 15H02213).

\section{References}

Couey JA, Marsh ER, Knapp BR, Ryan Vallance R., In-process force monitoring for precision grinding semiconductor silicon wafers, International Journal of Manufacturing Technology and Management, Vol.7, No.5/6 (2005), pp.430440.

Doi T, Uhlmann E, Marinescu ID., Handbook of Ceramics Grinding and Polishing (Second Edition), (2014), William Andrew.

Ebina Y, Maezaki T, Zhou L, Shimizu J, Onuki T, Ojima H and INUI M, Investigation of the Effect of Grain Size Variation on Ground Wafer Surface by Grinding Experiment/Simulation, Journal of the Japan Society for Precision Engineering, Vol.84, No.7 (2018), pp.640-45.

Gerhardt R. Properties and Applications of Silicon Carbide Edited by Rosario Gerhardt. (2011), DOI:10.1001/archotol.132.2.164.

Huang Ning, Yan Ying, Zhou Ping, Kang Renke, Guo Dongming, Saurav Goel., Elastic recovery of monocrystalline silicon during ultra-fine rotational grinding, Precision Engineering Vol.65, (2020), pp.64-71.

Ishibashi K, Tsukii Y, Ebina Y, Zhou L, Shimizu J, Yamamoto T, et al., Study on mechanism of rotary in-feed face 
grinding : Development of wireless dynamometer for rotary infeed face grinding, Journal of the Japan Society for Abrasive Technology, Vol.63, No.1 (2019), pp.31-35.

King, Robert I. and Hahn Robert S., Handbook of Modern Grinding Technology, (1986), Springer.

Lin B, Zhou P, Wang Z, Yan Y, Renke Kang DG., Analytical elastic plastic cutting model for predicting grain depth-ofcut in ultrafine grinding of silicon wafer, Journal of Manufacturing Science and Engineering, Vol. 140, No. 12 (2018), Paper No. MANU-18-1118.

Liu Y, Warkentin A, Bauer R, Gong Y., Investigation of different grain shapes and dressing to predict surface roughness in grinding using kinematic simulations, Precision Engineering, Vol.37, No.3 (2013), pp.758-764.

Lundt H, Kerstan M, Huber A, Hahn PO., Subsurface damage of abraded silicon wafers, Semiconductor Silicon/1994: Proceedings of the 7th International Symposium on Silicon Materials Science and Technology, (1994), pp.218-224.

Ono K., Grinding finishing., Maki-syoten, (1962), pp.80 (in Japanese).

Pei ZJ, Billingsley SR, Miura S., Grinding induced subsurface cracks in silicon wafers, International Journal of Machine Tools and Manufacture, Vol.39, No.7 (1999), pp.1103-1116.

Pishchik V, Lytvynov LA, Dobrovinskaya ER, Dobrovinskaya ER, Lytvynov LA, Pishchik V., Application of Sapphire, (2009), DOI:10.1007/978-0-387-85695-7_1.

Strukov BA, Levanyuk AP., Ferroelectric Phenomena in Crystals, (1998), DOI:10.1007/978-3-642-60293-1.

Sun W, Pei ZJ, Fisher GR., Fine grinding of silicon wafers: Machine configurations for spindle angle adjustments, International Journal of Machine Tools and Manufacture, Vol.45, No.1 (2005), pp.51-61.

Sun J, Qin F, Chen P, An T., A predictive model of grinding force in silicon wafer self-rotating grinding, International Journal of Machine Tools and Manufacture, Vol.109, No.10 (2016), pp.74-86.

Syoji K, Grinding Engineering, Yokendo Co., Ltd., (2004), pp.69 (in Japanese).

Tönshoff HK, v. Schmieden W, Inasaki I, König W, Spur G., Abrasive Machining of Silicon, CIRP Annals Manufacturing Technology, Vol.39, No.2 (1990), pp.621-635.

Tomita Y, Eda H., A study of the ultra precision grinding process on a magnetic disk substrate - Development of new bonding materials for fixed abrasives of grinding stone, Wear, Vol.195, No.1-2 (1996), pp.74-80.

Tso PL, Teng CC., A study of the total thickness variation in the grinding of ultra-precision substrates, Journal of Materials Processing Technology, Vol.116, No.2 (2001), pp.182-188.

Young HT, Liao HT, Huang HY., Surface integrity of silicon wafers in ultra precision machining, International Journal of Advanced Manufacturing Technology, Vol.29, No.3 (2006), pp.372-378.

Young HT, Liao HT, Huang HY., Novel method to investigate the critical depth of cut of ground silicon wafer, Journal of Materials Processing Technology, Vol.182, No.1-3 (2007), pp.157-162.

Zhang XH, Pei ZJ, Fisher GR., A grinding-based manufacturing method for silicon wafers: Generation mechanisms of central dimples on ground wafers, International Journal of Machine Tools and Manufacture, Vol.46, No.3-4 (2006), pp.397-403.

Zhang L, Pei C, Tong A, Dai Y, Fei Q., Analytical prediction for depth of subsurface damage in silicon wafer due to selfrotating grinding process, Current Applied Physics, Vol.19, No.5 (2019), pp.570-581.

Zhou L, Shimizu J, Shinohara K, Eda H., Three-dimensional kinematical analyses for surface grinding of large scale substrate, Vol.27, No.2 (2003), pp.175-184.

Zhou L, Tian YB, Huang H, Sato H, Shimizu J., A study on the diamond grinding of ultra-thin silicon wafers, Proceedings of the Institution of Mechanical Engineers, Part B: Journal of Engineering Manufacture, Vol.226, (2012), pp.66-75.

Zhou L, Ebina Y, Wu K, Shimizu J, Onuki T, Ojima H., Theoretical analysis on effects of grain size variation, Precision Engineering, Vol.50, (2017), pp.27-31. 